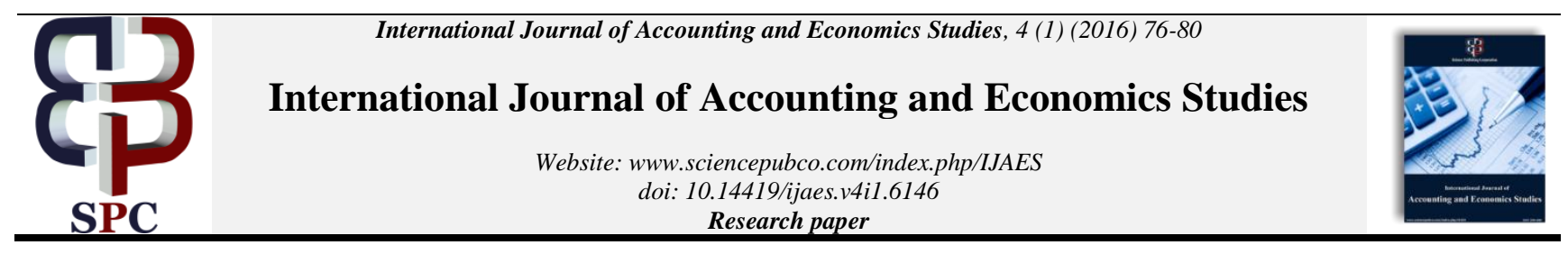

\title{
Estimating the probability of forecasted events
}

\author{
Alexander Madera* \\ National Research University Higher School of Economics 20 Myasnitskaya Str., Moscow 101000 Russia \\ *Corresponding author E-mail: agmprof@gmail.com
}

\begin{abstract}
The article elaborates a method for estimating the probabilities of occurrence of prognosticated events in future. On the basis of the data from the previous periods about prognosticating the relevant events, as well as the data about the trends observed at present, two matrices are formed, the product of which is the matrix for the prognosis errors committed by the individual or the expert. The article shows that the vector for probabilities of the prognosticated events is the eigenvector of the prognosis error matrix, which corresponds to its single eigenvalue. Application of the elaborated method is shown on the definite example for forecasting demand of new products.
\end{abstract}

Keywords: Events; Eigenvector; Eigenvalue; Forecast; Probability.

\section{Introduction}

Individual decision making is significantly determined by the uncertainty of future outcomes and consequences that will take place after an individual makes the decision and implements it into practice. When making a decision, an individual may generate many possible consequences and scenarios and each of them can be implemented in future. However, it cannot be said a priori which event will take place and which scenario will be implemented. Uncertainty of the future, consequences, and outcomes of the decisions is the key obstacle for making the optimal decision by an individual in terms of achieving the goal. Indeed, the optimal decision made by an individual (in his/her opinion) is optimal only for the today's reality; however, in tomorrow's actuality (after a week, a month, a year, or several years), not only may the decision turn out to be not optimal but it also may cause quite opposite results from what was expected due to the irreversible and constant changes both in internal and internal conditions.

Any prediction of future events is subjective, since is made by a party (a person making the decision; an expert, either individual or a group) whose abilities to predict future events and their probability are rather limited (Kahneman et al. 2010, Madera 2014 a, Madera 2014 b, Madera 2015). In this connection, the methods for scientific prediction of events and their probabilities for different time horizons have become especially topical. These methods need to take into account both the relevant information that is obtained at the present moment and the data referring to prediction of relevant events and their implementation in the past (Clauset \& Woodard 2013, Clemen 1989, Mohler 2013, Rossi 2010).

Most existing methods for predicting events and their probability are based on an assumption that the past and future are indiscernible, so the past trends will be the same in the future. Extrapolation of the previous data onto future is used as a prediction method. This principle underlies regressions, time series, various types of moving averages, smoothing, etc. (Green 2012, Hanke et al. 2001, McNees 1990, Mirkin 2014, Rossi 2010). This prediction conception indicates that people tend to underestimate, or in many cases even neglect, uncertainty of future (Madera 2014 a, Makridakis 1986). A number of methods for predicting financial and commodity markets employ the hypothesis that the financial tools (stock price, currency exchange rates, prices for raw materials, fuel, real estate, etc.) are stochastic despite the fact that this hypothesis has not been proven in practice. Furthermore, numerous facts demonstrate that a price change cannot be predicted based on the historical time series of price changes over the past periods (Mantegna \& Stanley 2000); there may be several-fold deviation between the predicted prices and the actual ones for all the types of raw materials, stock prices, and currency exchange rates (Mirkin 2014). Expert evaluations, which are inevitably subjective, are used when the data on predicting relevant events over the past periods are either unavailable or insufficient for the analysis (Armstrong 2001, Green 2012, Hanke et al. 2001). In many studies, when assessing the probabilities of future events, the researchers build regression models (Haan \& Sinha 1999), describe the events as Markov random processes (Boer et al. 2007), or assume a priori that a random phenomenon under study obeys a certain law of probability distribution (typically, the normal probability distribution) (Clauset \& Woodard 2013, Mohler 2013, Riley 2012).

In the classical approach the probabilities of occurrence of prognosticated events in future are assessed, as a rule, using Bayes' theorem, according to which the a priori probabilities of the prognosticated events are transformed into the a posteriori probabilities based on the new information obtained as a result of conducting an experiment. Otherwise speaking, the a priori probabilities $p\left(A_{j}\right)$ of occurrence of the prognosticated events $A_{j}, j=$ $1,2, \ldots, n$, that are exhaustive are transformed according to Bayes' theorem into the a posteriori probabilities $p\left(A_{j} \mid B\right)$ with using

the data obtained after having conducted the experiment in which some event B occurs jointly with one of the prognosticated events. In order to apply Bayes' theorem, it is necessary to know the a priori probabilities $p\left(A_{j}\right), j=1,2, \ldots, n$, of the prognosticated 
events, as well as the conditional probabilities $p\left(B \mid A_{j}\right)$ determining the appearance of the event $B$ provided that each of the events $A_{j}$ occurs. In this situation, the probabilities $p\left(A_{j}\right)$ and $p\left(B \mid A_{j}\right), j=1,2, \ldots, n$, are a priori known only in a limited number of cases, which can be modeled with the use of urn-withballs type probabilistic models. At the same time, when considering events that pertain to human activities and their results (in economy, management, finance, decision-making, etc.), the probabilities of occurrence of future events cannot already be considered as known a priori, because man is not granted the capability of reliably predicting which specific events will occur in future and what probabilities of their occurrence will be. Due to this fact, the probabilities $p\left(A_{j}\right)$ of the prognosticated events $A_{j}, j=$ $1,2, \ldots, n$, to the same extent as the probabilities $p\left(B \mid A_{j}\right)$ of appearance of the event $B$ provided that the events $A_{j}$ occur in future, can be interpreted only as subjective probabilities, the values of which are assigned by the individual (expert) on the basis of his or her own understanding of the course of events and observed trends. The subjective nature determining the probabilities of the prognosticated events occurrence makes unreliable any forecasting with regard to man and their activities.

This article proposes a method for determining the a priori unknown probabilities $P(A)=\left(p\left(A_{1}\right), p\left(A_{2}\right), \ldots, p\left(A_{n}\right)\right)$ of occurrence of the prognosticated events $A_{1}, A_{2}, \ldots, A_{n}$ that makes it possible to reduce the amount of subjectivity when prognosticating. The method uses two types of data: data related to prognosticating the relevant events from periods in the past and data about the trends observed at present, which, in the individual's opinion, can cause occurrence of certain events in future. According to these data, the matrix $(L)$ for errors of prognosticating the relevant events in the previous periods and the matrix $(M)$ for the adjusted prognoses based on the present information are arranged. The article shows that the vector $P(A)=\left(p\left(A_{1}\right), p\left(A_{2}\right), \ldots, p\left(A_{n}\right)\right)$

is the eigenvector of the prognosis error matrix $K=M \cdot L$, which corresponds to the single eigenvalue of the matrix $K$. Because the probability values for the prognosticated events are elements of the eigenvector $P(A)$ of the matrix $K$, the calculation method for which does not depend on the individual, the proposed method, as opposed to Bayesian approach, makes it possible to reduce the subjectivity when determining the probabilities of the prognosticated events. The article gives an example of using the developed method.

\section{Event prognosis errors in previous periods and at present}

When prognosticating the occurrence of future events $A_{j}, j=$ $1,2, \ldots, n$, which are exhaustive (the set $\mathbf{A}$ ), the individual relies on the observations of the other events $B_{i}, i=1,2, \ldots, n$, which are also exhaustive (the set $\mathbf{B}$ ). The events $A_{1}, A_{2}, \ldots, A_{n} \in \mathbf{A}$ will further be called the real events, and the events $B_{1}, B_{2}, \ldots, B n$

$\in \mathbf{B}$ will be the prognosticating events.

It is obvious that none of the events observed in the past or at present can evidence unambiguously and absolutely reliably the occurrence of any event in the future reality. That is why occurrence of a certain real event $A_{j} \in \mathbf{A}$ based on the occurrence of certain prognosticating events $B_{i} \in \mathbf{B}$ is always prognosticated with some error that reflects the individual's (expert's) prognosis mistakes. As a result of this, the event $B_{i} \in \mathbf{B}(i=1,2, \ldots, n)$ that prognosticates, in the individual's opinion, occurrence of the real event $A_{j} \in \mathbf{A}$ can appear with any of the events

${ }^{A_{1}}, A_{2}, \ldots, A_{n} \in \mathbf{A}$, i. e. $B_{i}=\bigcup_{k} B_{i} A_{k}$.

The individual's (expert's) prognosis error values are determined by the following conditional probabilities $(i, j=1,2, \ldots, n)$ :

- $p\left(B_{i} \mid A_{j}\right)$ is the conditional probability of occurrence of the prognosticating event $B_{i}$, provided that one of the real events $A_{j} \in \mathbf{A}$ has occurred, indeed. This error is determined by the statistical data about the individual's (expert's) prognosticating the relevant events in the previous periods and shows the relative quantity of both correct and incorrect prognoses;

- $p\left(A_{j} \mid B_{i}\right)$ is the conditional probability of future occurrence of the real event $A_{j}$, provided that the prognosticating event $B_{i}$ $\in \mathbf{B}$ is occurring at present. The value of the probability $p\left(A_{j} \mid B_{i}\right)$ is the prognosis error and reflects the degree of the individual's belief in occurrence of the real events $A_{j} \in \mathbf{A}(j=1$, $2, \ldots, n)$, if a certain prognosticating event $B_{i} \in \mathbf{B}$ occurs in future. It is obvious that the values of the errors $p\left(A_{j} \mid B_{i}\right), i, j=$ $1,2, \ldots, n$, can become known only when "the future" comes and the real event $A_{j} \in \mathbf{A}(j=1,2, \ldots, n)$ happens in it.

Having determined, according to the statistical data from the previous periods, the prognosis error values $p\left(B_{i} \mid A_{j}\right), i, j=$

$1,2, \ldots, n$, we will receive, using the total probability formula, a system of $\mathrm{n}$ equalities that determine the total probabilities $p\left(B_{i}\right)$ of occurrence of the prognosticating events $B_{i} \quad(i=$ $1,2, \ldots, n)$ :

$p\left(B_{i}\right)=\sum_{j=1}^{n} p\left(A_{j}\right) p\left(B_{i} \mid A_{j}\right), i=1,2, \ldots, n$.

With the $\mathrm{n}$ column vectors for the probabilities of the prognosticating events $P(B)=\left(p\left(B_{1}\right), p\left(B_{2}\right), \ldots, p\left(B_{n}\right)\right)^{T},\left((\cdot)^{T}\right.$ is the transposition operation) and yet unknown probabilities of the real events $P(A)=\left(p\left(A_{1}\right), p\left(A_{2}\right), \ldots, p\left(A_{n}\right)\right)^{T}$, as well as the $n \times n$ matrix $L=\left\|p\left(B_{i} \mid A_{j}\right)\right\|$ for the prognosis errors in the previous periods having been introduced,

$$
L=\left(\begin{array}{cccc}
p\left(B_{1} \mid A_{1}\right) & p\left(B_{1} \mid A_{2}\right) & \cdots & p\left(B_{1} \mid A_{n}\right) \\
p\left(B_{2} \mid A_{1}\right) & p\left(B_{2} \mid A_{2}\right) & \cdots & p\left(B_{2} \mid A_{n}\right) \\
\vdots & \vdots & \cdots & \vdots \\
p\left(B_{n} \mid A_{1}\right) & p\left(B_{n} \mid A_{2}\right) & \cdots & p\left(B_{n} \mid A_{n}\right)
\end{array}\right),
$$

The system of equations (1) may be written as a matrix:

$$
P(B)=L \cdot P(A) .
$$

When prognosticating the events and assessing the probabilities of their occurrence, the individual is guided by not only the prognosis data from the previous periods, but also by the new information about the events and trends observed at present. By relying on the obtained data, the individual estimates the probabilities $p\left(A_{j} \mid B_{i}\right), i, j=1,2, \ldots, n$, of occurrence of the real events $A_{j}$ $\in \mathbf{A}(j=1,2, \ldots, n)$ on the basis of his or her own (or the expert's) 
beliefs regarding the influence that each of the prognosticating events $B_{i} \in \mathbf{B}(i=1,2, \ldots, n)$ exerts on the occurrence of each real event $A_{j} \in \mathbf{A}(j=1,2, \ldots, n)$.

Because each real event $A_{j} \in \mathbf{A}(j=1,2, \ldots, n)$ can appear jointly with any prognosticating event $B_{i} \in \mathbf{B}$, i. e. $A_{j}=\bigcup_{k} A_{j} B_{k}$, according to the total probability formula we can write a system of $\mathrm{n}$ equalities to determine the total probabilities $p\left(A_{j}\right)$ of occurrence of the prognosticated real events $A_{j}(j=1,2, \ldots, n)$ :

$$
p\left(A_{j}\right)=\sum_{i=1}^{n} p\left(B_{i}\right) p\left(A_{j} \mid B_{i}\right), j=1,2, \ldots, n .
$$

With the $n \times n$ matrix $M=\left\|p\left(A_{j} \mid B_{i}\right)\right\|$ for the prognosis errors at present having been introduced

$M=\left(\begin{array}{cccc}p\left(A_{1} \mid B_{1}\right) & p\left(A_{1} \mid B_{2}\right) & \cdots & p\left(A_{1} \mid B_{n}\right) \\ p\left(A_{2} \mid B_{1}\right) & p\left(A_{2} \mid B_{2}\right) & \cdots & p\left(A_{2} \mid B_{n}\right) \\ \vdots & \vdots & \cdots & \vdots \\ p\left(A_{n} \mid B_{1}\right) & p\left(A_{n} \mid B_{2}\right) & \cdots & p\left(A_{n} \mid B_{n}\right)\end{array}\right)$,

The system of the equations (4) may be written as a matrix:

$$
P(A)=M \cdot P(B) .
$$

The elements of the matrices $L, M$ and vectors $P(A)$ and $P(B)$ are nonnegative and satisfy the following equalities:

$$
\begin{aligned}
& \sum_{i=1}^{n} p\left(B_{i} \mid A_{j}\right)=1, \sum_{j=1}^{n} p\left(A_{j} \mid B_{i}\right)=1(\mathrm{i}, \mathrm{j}=1,2 \ldots \mathrm{n}), \\
& \sum_{j=1}^{n} p\left(A_{j}\right)=1, \sum_{i=1}^{n} p\left(B_{i}\right)=1 .
\end{aligned}
$$

A nonnegative (positive) matrix where the sum of the elements in each column equals +1 is called, as it is known, a nonnegative (positive) stochastic matrix, and a nonnegative (positive) vector where the sum of the elements equals +1 is called a probability vector.

\section{Determining the probabilities of the fore- casted events}

By substituting, in the right part of equality (6), the expression of vector $P(B)$ from equality (3) for the vector itself, we will obtain an equation for determining the sought vector for the probabilities of the prognosticated real events $P(A)$ :

$$
P(A)=K \cdot P(A),
$$

where $K=M \cdot L=\left\|k_{i j}\right\|$ is an $n \times n$ matrix.

As it follows from equation (7), the vector of the probabilities $P(A)=\left(p\left(A_{1}\right), p\left(A_{2}\right), \ldots, p\left(A_{n}\right)\right)^{T}$ of occurrence of the real events $A_{j} \in \mathrm{A}, j=1,2, \ldots, n$, is the eigenvector of the stochastic matrix $K$, which corresponds to its single eigenvalue.
It is possible to prove that the matrix $K=M \cdot L=\left\|k_{i j}\right\|$ has the following important properties (Bellman 1960, Horn \& Johnson 2013):

1) The product of two stochastic matrices is again a stochastic matrix, and the product of a stochastic matrix and a probability vector is a probability vector.

2) If $A \geq 0$ is a nonnegative matrix, the spectral radius $\rho(A)$ of the matrix $\mathrm{A}$ is its eigenvalue and there is a nonnegative vector $x \geq 0, x \neq 0$ for which $A x=\rho(A) x$.

3) If the column totals are constant for the nonnegative matrix A, the spectral radius of the matrix equals $\rho(A)=\|A\|_{1}$, where $\|A\|_{1}$ is the maximal column norm, which is determined by the formula

$$
\|A\|_{1}=\max _{1 \leq j \leq n} \sum_{i=1}^{n} a_{i j} .
$$

4) For the indecomposable and nonnegative matrix $A$, the value $\rho(A)$ is an algebraically simple (not multiple) eigenvalue for $A$.

Let us note that the stochastic matrix can always be represented as a positive matrix $(A>0)$, for which the assertions of the given properties are automatically fulfilled.

As it follows from the properties given:

a) The matrix $K=M \cdot L$ is stochastic and the product of the matrix $K$ and the probability vector $P(A)$ in equation (7) is a probability vector;

b) The maximal eigenvalue $\lambda$ of the stochastic matrix $K$ equals +1 , and the single probability eigenvector $P(A)$ corresponds to it.

As the matrix $L$ comprises information about the prognosis errors in the previous periods, and the matrix $M$ does the same with the present prognosis errors, the matrix $K=M \cdot L$ characterizes the total prognosis error intrinsic to the individual (expert) when drawing up the prognoses. Let us note that the prognosis errors depend both on the individual (expert) who draws up the prognoses and on the problem regarding which the prognosis is made, and in this situation the prognosis data from the previous periods must be valid and relevant to the problem considered.

Matrix equation (7) may be written as a system of $n$ linear algebraic equations, and for it to determine unambiguously the eigenvector of the matrix $K$, it is necessary to supplement it with the normalizing equality $\sum_{j=1}^{n} p\left(A_{j}\right)=1$. Thus, the system of the equations that determines unambiguously the $\mathrm{n}$ sought probabilities $p\left(A_{i}\right)$, $i=1,2, \ldots, n$ of occurrence of the prognosticated real events $A_{i} \in$ A, $i=1,2, \ldots, n$ will look as follows:

$$
p\left(A_{i}\right)=\sum_{j=1}^{n} k_{i j} \cdot p\left(A_{i}\right), i=1,2, \ldots, n,
$$

$$
\sum_{j=1}^{n} p\left(A_{j}\right)=1
$$

\section{An example of applying the method}

Let us consider the following practical situation. A manufacturing company is planning to launch a new product, and in order to make the final decision, the company management draws up a prognosis of its future demand $(D)$. In the opinion of the management, the demand for the new product can have three levels: a 
high one $\left(D_{h}\right)$, an average one $\left(D_{m}\right)$ and a low one $\left(D_{l}\right)$. Let us suppose that the company has the data from the previous periods about prognosticating the demand for similar products manufactured earlier and the demand levels that have been implemented in practice.

The data from the past show that when the market research predicted a high level of the demand $\left(D_{h, p r}\right)$ for the new product, in $62 \%$ of the cases the prognosis was correct and the high demand level $\left(D_{h}\right)$ was really observed, i. e. $p\left(D_{h, p r} \mid D_{h}\right)=0.62$.

However, in $22 \%$ and $17 \%$ of the cases when the market research predicted the high demand level, the real demand was average $\left(D_{m}\right)$ or even low $\left(D_{l}\right)$ in reality, that is why $p\left(D_{h, p r} \mid D_{m}\right)$ $=0.22$ and $p\left(D_{h, p r} \mid D_{l}\right)=0.17$. Similar data from the previous periods about prognosticating the average $\left(D_{m}, p r\right)$ and low $\left(D_{l, p r}\right)$ levels of the demand for the new products, as well as the data about the demand levels that have been implemented in practice make it possible to determine the percentages of both correct and incorrect prognoses and calculate the prognosis errors for the previous periods. The results of the calculations are given in the error matrix $L$ :

$$
\begin{aligned}
& L=\left(\begin{array}{ccc}
p\left(D_{h, p r} \mid D_{h}\right) & p\left(D_{h, p r} \mid D_{m}\right) & p\left(D_{h, p r} \mid D_{l}\right) \\
p\left(D_{m, p r} \mid D_{h}\right) & p\left(D_{m, p r} \mid D_{m}\right) & p\left(D_{m, p r} \mid D_{l}\right) \\
p\left(D_{l, p r} \mid D_{h}\right) & p\left(D_{l, p r} \mid D_{m}\right) & p\left(D_{l, p r} \mid D_{l}\right)
\end{array}\right)= \\
& =\left(\begin{array}{lll}
0,62 & 0,22 & 0,17 \\
0,15 & 0,74 & 0,25 \\
0,23 & 0,04 & 0,58
\end{array}\right) .
\end{aligned}
$$

Along with this, the market situation that has been forming by now does not make it possible to draw firm conclusions about the future level of the demand for the new product. Based on the new data, the management of the company thinks that if the trend leading to the high demand level ( $D_{h, p r}$ ) turns into reality, it is possible to expect in $43 \%$ of the cases that the demand will actually be high $\left(D_{h}\right)$, i. e. $p\left(D_{h} \mid D_{h}, p r\right)=0.43$. However, taking into account that the ambiguity of the information received, the direction thinks that the situation can change, and, if there is the high demand tendency, the average level of demand $\left(D_{m}\right)$ can realize in $24 \%$ of the cases and the low demand level $\left(D_{l}\right)$ can do so in $33 \%$ of the cases, that is why $p\left(D_{m} \mid D_{h}, p r\right)=0.24$ and $p\left(D_{l} \mid D_{h, p r}\right)=0.33$. Similar conclusions made on the basis of the new data lead to the following prognosis error matrix M:

$M=\left(\begin{array}{ccc}p\left(D_{h} \mid D_{h, p r}\right) & p\left(D_{h} \mid D_{m, p r}\right) & p\left(D_{h} \mid D_{l, p r}\right) \\ p\left(D_{m} \mid D_{h, p r}\right) & p\left(D_{m} \mid D_{m, p r}\right) & p\left(D_{m} \mid D_{l, p r}\right) \\ p\left(D_{l} \mid D_{h, p r}\right) & p\left(D_{l} \mid D_{m, p r}\right) & p\left(D_{l} \mid D_{l, p r}\right)\end{array}\right)=$

$$
=\left(\begin{array}{lll}
0,43 & 0,18 & 0,07 \\
0,24 & 0,57 & 0,14 \\
0,33 & 0,25 & 0,79
\end{array}\right) .
$$

Then the matrix for the total prognosis error $K=M \cdot L$ equals

$$
K=M \cdot L=\left(\begin{array}{lll}
0,309 & 0,231 & 0,158 \\
0,267 & 0,480 & 0,265 \\
0,424 & 0,289 & 0,577
\end{array}\right)
$$

The vector for the sought probabilities $p(D)=\left(p\left(D_{h}\right), p\left(D_{m}\right), p\left(D_{l}\right)\right)$ of the prognosticated demand levels is the eigenvector of the matrix $K$ and is determined from the matrix equation $P(D)=K \cdot P(D)$ with adding the normalizing equality to it. As one of the equations in the system of the three equations that follow from $P(D)=K \cdot P(D)$ depends linearly on the two others, one of them should be deleted (any of them, generally speaking). Having rejected, for example, the third equation, we will obtain the following system of the equations, the solution of which determines unambiguously the probabilities of the prognosticated demand levels $p\left(D_{h}\right), p\left(D_{m}\right), p\left(D_{l}\right)$ :

$$
\begin{aligned}
& 0,309 \cdot p\left(D_{h}\right)+0,231 \cdot p\left(D_{m}\right)+0,158 \cdot p\left(D_{l}\right)=p\left(D_{h}\right), \\
& 0,267 \cdot p\left(D_{h}\right)+0,480 \cdot p\left(D_{m}\right)+0,265 \cdot p\left(D_{l}\right)=p\left(D_{m}\right), \\
& p\left(D_{h}\right)+p\left(D_{m}\right)+p\left(D_{l}\right)=1 .
\end{aligned}
$$

The solution of this system of the equations is equal to $p\left(D_{h}\right)$ $=0.215 ; p\left(D_{m}\right)=0.338 ; p\left(D_{l}\right)=0.447$. The obtained values of the probabilities make it possible to recommend the company management to make the decision that corresponds to the maximal probability of realization of the demand for the new product in future. As the probability of the actual future occurrence of the low demand level $(=0.447)$ is higher than the probabilities of the high $(=0.215)$ or average $(=0.338)$ demand levels, the new product should not be produced.

\section{Conclusion}

The method elaborated by the article makes it possible to prognosticate future events and assess the probabilities of their occurrence. The probabilities of the prognosticated events are coordinated with two types of data obtained from both previous periods when the relevant events were prognosticated and the tendencies observed at present. Based on these data, two matrices are formed, one of which is the matrix of the prognosis errors in the previous periods $(L)$ and the second one is the matrix for the errors of the prognoses $(M)$ drawn up on the basis of the new information. The product of the matrices $K=M \cdot L$ contains the complete information about the errors intrinsic to the individual (expert) when drawing up the prognoses. The article shows that the sought vector for the probabilities of occurrence of the prognosticated events in future is the eigenvector of the matrix $K$, which corresponds to its single eigenvalue. The obtained probabilities of the prognosticated events make it possible to increase adequacy of prognosticating the future events and reduce its subjective constituent.

\section{References}

[1] Armstrong JS, ed. (2001) Principles of Forecasting: A Handbook for Researchers and Practitioners, Kluwer. http://dx.doi.org/10.1007/978-0-306-47630-3.

[2] Bellman R (1960) Introduction to Matrix Analysis, McGraw-Hill Book Company Inc.

[3] Boer P.T, Ecuyer P, Rubino G, Tuffin B. Estimating the probability of a rare event over a finnite time horizon (2007). Proceedings of the 2007 Winter Simulation Conference 403-411. http://dx.doi.org/10.1109/WSC.2007.4419629.

[4] Clauset A, Woodard R (2013) Estimating the histirical and future probabilities of the large terrorist events. The Annals of Applied Statistics 7, 1838-1865. http://dx.doi.org/10.1214/12-AOAS614

[5] Clemen R (1989) Combining Forecast. International Journal of Forecasting 5, 559-583. http://dx.doi.org/10.1016/01692070(89)90012-5.

[6] Green WH (2012) Econometric Analysis. Pearson, Boston. 
[7] Haan L, Sinha AK (1999) Estimating the probability of a rare event. The Annals of Statistics 27, 732-759. http://dx.doi.org/10.1214/aos/1018031214.

[8] Hanke JE, Reitsch AG, Wichern DW (2001) Business Forecasting. Prentice Hall Inc., N.J.

[9] Horn RA, Johnson CR (2013) Matrix Analysis. Cambridge University Press, N.Y.

[10] Kahneman D, Slovic P, Tversky A (2001) Judgment under Uncertainty: Heuristics and Biases. Cambridge University Press, Cambridge.

[11] Madera AG (2014 a) Risks and Chances: Uncertainty, Forecasting and Evaluation. Krasand, Moscow.

[12] Madera AG (2014 $b$ ) Interval stochastic uncertainty of estimates in multiple criteria decision making problems. Artificial Intelligence and Decision Making 3, 105-115.

[13] Madera AG (2015) Interval Uncertainty of estimates and judgments of subject in decision making in multi-criteria problems. International Journal of the Analytic Hierarchy Process 7, 337-348.

[14] Makridakis S. (1986) the art and science of forecasting. International Journal of Forecasting 2, 15-39. http://dx.doi.org/10.1016/0169-2070(86)90028-2.

[15] Mantegna RN, Stanley HE (2000) an Introduction to Econophysics. Correlations and Complexity in Finance. Cambridge University Press, Cambridge

[16] McNees SK (1990) the role of judgment in macroeconomic forecasting accuracy. International Journal of Forecasting 6, 287-299. http://dx.doi.org/10.1016/0169-2070(90)90056-H.

[17] Mirkin J ed (2014) International Practice of Forecasting World Prices in the Financial Markets (Raw Materials, Stocks, Exchange Rates). Magistr, Moscow.

[18] Mohler G (2013) Discussion of "Estimating the historical and future probabilities of the large terrorist events" by Aaron Clauset and Ryan Woodard. The Annals of Applied Statistics 7, 1866-1870. http://dx.doi.org/10.1214/13-AOAS614A.

[19] Riley P (2012) on the probability of occurrence of extreme space weather events. Space Weather 10, $12 \mathrm{p}$.

[20] Rossi E (2010) Univariate GARCH models: A Survey. Quantile 8 , 1-67. 